Article

\title{
Low-Cost Photolithographic Fabrication of Nanowires and Microfilters for Advanced Bioassay Devices
}

\author{
Nhi M. Doan ${ }^{1, \dagger}$, Liangliang Qiang ${ }^{1, \dagger}$, Zhe Li ${ }^{1}$, Santhisagar Vaddiraju ${ }^{1,2}$, Gregory W. Bishop ${ }^{3}$, \\ James F. Rusling ${ }^{3,4,5,6}$ and Fotios Papadimitrakopoulos $1,3,4, *$
}

1 Nanomaterials Optoelectronics Laboratory, Polymer Program, University of Connecticut, Storrs, CT 06269, USA; E-Mails: nhi.doan@uconn.edu (N.M.D.); liangliang.qiang@gmail.com (L.Q.); zhe.li@uconn.edu (Z.L.); sagar@bio-orasis.com (S.V.)

2 Biorasis Inc., 23 Fellen Road, Storrs, CT 06268, USA

3 Department of Chemistry, University of Connecticut, Storrs, CT 06269, USA; E-Mails: greg.bishop@uconn.edu (G.W.B.); james.rusling@uconn.edu (J.F.R.)

4 Institute of Materials Science, University of Connecticut, Storrs, CT 06269, USA

5 Department of Cell Biology, University of Connecticut Health Center, Farmington, CT 062032, USA

6 School of Chemistry, National University of Ireland, Galway, Ireland

$\dagger$ These authors contributed equally to this work.

* Author to whom correspondence should be addressed; E-Mail: papadim@ims.uconn.edu; Tel.: +8-60-486-3447; Fax: +8-60-486-4745.

Academic Editor: Kagan Kerman

Received: 14 January 2015 / Accepted: 2 March 2015 / Published: 12 March 2015

\begin{abstract}
Integrated microfluidic devices with nanosized array electrodes and microfiltration capabilities can greatly increase sensitivity and enhance automation in immunoassay devices. In this contribution, we utilize the edge-patterning method of thin aluminum (Al) films in order to form nano- to micron-sized gaps. Evaporation of high work-function metals (i.e., $\mathrm{Au}, \mathrm{Ag}$, etc.) on these gaps, followed by $\mathrm{Al}$ lift-off, enables the formation of electrical uniform nanowires from low-cost, plastic-based, photomasks. By replacing Al with chromium $(\mathrm{Cr})$, the formation of high resolution, custom-made photomasks that are ideal for low-cost fabrication of a plurality of array devices were realized. To demonstrate the feasibility of such $\mathrm{Cr}$ photomasks, SU-8 micro-pillar masters were formed and replicated into PDMS to produce micron-sized filters with 3-4 $\mu \mathrm{m}$ gaps and an aspect ratio of 3 . These microfilters were capable of retaining $6 \mu \mathrm{m}$ beads within a localized site, while allowing solvent flow. The combination of nanowire arrays and micro-pillar filtration opens new perspectives for
\end{abstract}


rapid R\&D screening of various microfluidic-based immunoassay geometries, where analyte pre-concentration and highly sensitive, electrochemical detection can be readily co-localized.

Keywords: nanowire; nanogaps; microelectrode arrays; sensor; immunoassay; microfluidic; photolithography; isotropic etching

\section{Introduction}

Integrated microfluidic devices have shown significant promise in lowering cost and increasing automation of immunoassay detection [1,2]. A variety of detection methods including amperometry [3-8], electrochemiluminescene [9-12], field-effect transistor (FET) [13-18], optical sensing [16,19-21], etc. have been incorporated within microfluidic devices. Some microfluidic immunoassay devices, have been also outfitted with online micro-filters to pre-concentrate the antigen of interest $[19,22,23]$ or filter out blood platelets [24]. Typical microfilters are composed of large aspect-ratio trenches or micropillars with sub-micron gaps [22,25,26]. Microfilters have been fabricated by a number of sophisticated techniques involving electron-beam-lithography [27-29], deep-reactive ion etching [22], nanoimprinting [28,30], and dip-pen nanolithography [31]. The inherent expenses associated with these fabrication methodologies naturally inflate $R \& D$ costs, where multiple iterations are needed to optimize a certain device configuration. The ingenious "edge patterning" technique of Whitesides et al. [32], have enabled the formation of nano- to sub-micron sized features using traditional photolithography and isotropic etching of a reactive metal (i.e., aluminum). As shown in Figure 1, metal undercutting beneath the photoresist edge results in nanosized trenches with widths of $c a .50 \mathrm{~nm}$. The widths of these trenches are typically limited by the grain-size inhomogeneity of the deposited metal film [32].

In this contribution, we describe the use of edge-patterning method to produce nanowire-based array electrodes along with high-aspect ratio microfilters that can be both integrated within PDMS (polydimethylsiloxane)-based microfluidics. The latter approach enables selective localization and pre-concentration of micron-sized beads at predetermined locations, which is important for electrochemical-based immunodetection [23]. In this manner, a number of signal amplification techniques can be implemented, as a consequence of the high surface-to-volume ratio that these microbeads provide [8,22,23,33-35], alongside the facile incorporation of large number of enzyme labels, (e.g., horseradish peroxidase, glucose oxidase, etc.) compared to capturing antibodies $[8,36]$. While magnetic microbeads can be easily localized using magnetic fields, micropillar-based filters extend such localization to non-magnetic beads as well. With a typical size of microbead diameter between 1 and $10 \mu \mathrm{m}$, fabrication of high-aspect ratio micropillar filters using traditional photo-lithography is a challenging task that requires the use of fairly expensive, high-end photolithography masks [25]. We herein show that high resolution, custom-made masks can also be readily fabricated using the edge-patterning method. These are based on inexpensive plastic masks in combination with traditional UV photolithography or a handheld UV lamp to achieve gaps of $c a .4$ microns and height-to-width aspect ratio of 3. Additionally the as-produced edge-patterned gaps can be used to define micron- to nano-sized wires for microelectrode arrays [37]. Such microelectrode arrays can significantly amplify electrochemical signals due to the radial mass transport of electroactive species towards the sensor electrode [38]. 
The use of edge-patterning method to co-localize nanowire arrays and micro-pillar filtration opens new capabilities for the facile production of advanced microfluidic devices that could ultimately lower the cost and increase automation for electrochemical immunoassays [39].
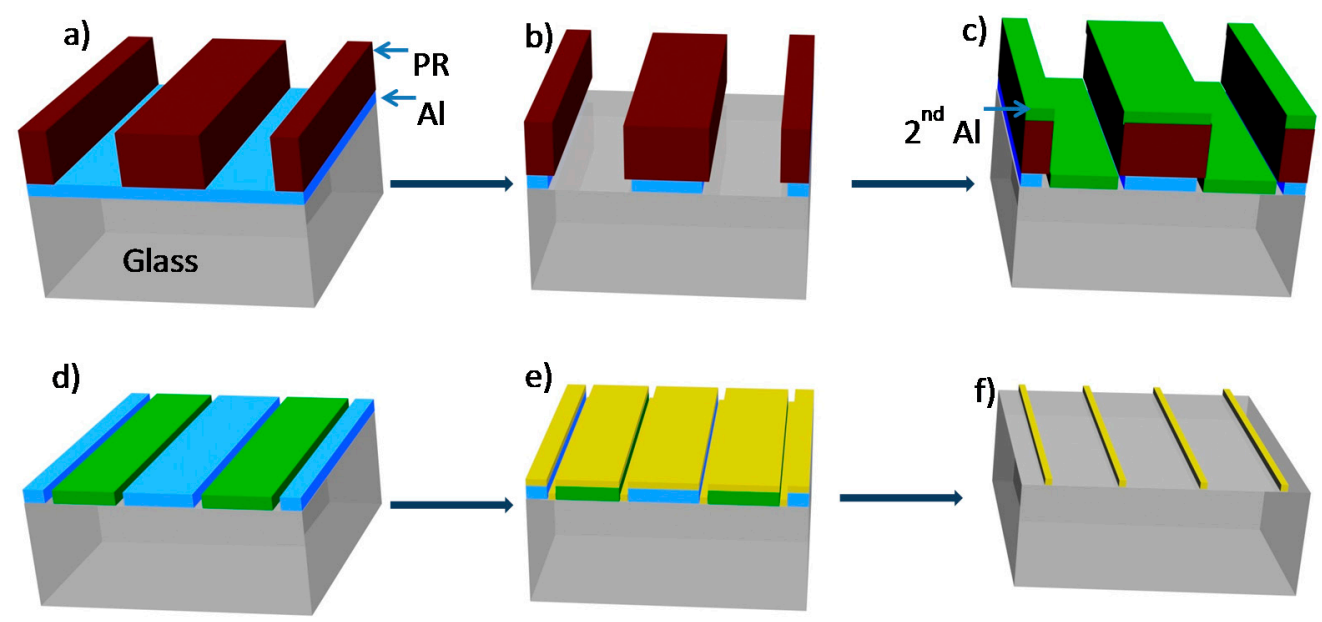

Figure 1. "Edge-patterning" of a thin aluminum film to realize nano- and micron-sized gap and wires. The process starts via photoresist (PR) patterning (a); isotropic etch to realize $\mathrm{Al}$ undercut (b); evaporation of a 2nd Al layer (c); followed by photoresist lift-off to realize nano- and micron-sized gaps (d); Nano- and micron-sized wires are subsequently formed by depositing a higher work function metal onto the gaps (e); followed by aluminum lift-off (f).

\section{Experimental Section}

\subsection{Materials and Instrumentation}

All chemicals were procured from Aldrich (St. Louis, MO, USA). Gold (99.99\%), silver (99.99\%), aluminum (99.99\%), and chromium (99.99\%) were obtained from Kurt Lesker (Jefferson Hills, PA, USA). All glass substrates were Fisherfinest microscope slides from premium plain glass. Shipley S1813 photoresist, Developer-351, SU-8 3010, and SU-8 developer were purchased from Microchem Corp. (Newton, MA, USA). Sylgard 184 PDMS pre-polymer and corresponding curing agents were obtained from Dow Corning (Midland, MI, USA). Photolithography was carried out using a MA-6 mask aligner (Karl Süss, Sunnyvale, CA, USA). Scanning electron microscopic (SEM) images were collected by a JSM-6335F instrument (JEOL, Peabody, MA, USA) with a cold cathode field emission source at $10.0 \mathrm{kV}$ and working distance of $8 \mathrm{~mm}$.

\subsection{Nanogap Formation}

Nanowires and nanogaps were constructed on glass substrates, which were sequentially cleaned by sonicating in aqueous suspension of Micro-90 soap, then acetone, and then deionized (DI) water, for 30 min each. Following 4 min oxygen plasma treatment of these substrates (Plasma Gas System 210, PVA TePla America, Corona, CA, USA, Oxygen flow at $220 \mathrm{sccm}$, pressure $800 \mathrm{mTorr}, 400 \mathrm{~W}$ ), thermal evaporation of $\mathrm{Al}$ (or $\mathrm{Cr}$ ) metal ( $c a .100 \mathrm{~nm}$ ) was conducted on a Denton Vacuum (Moorestown, NJ, USA) DV 502A evaporator at a base pressure of $10^{-6}$ Torr and an average deposition 
rate of $10 \AA / \mathrm{s}$. Subsequently, $1.5 \mu \mathrm{m}$ of S1813 photoresist was spin-coated and baked at $120{ }^{\circ} \mathrm{C}$ for 10 min. Following UV exposure, development and stripping (using oxygen plasma), the exposed metal was over-etched in a mixture of $\mathrm{H}_{3} \mathrm{PO}_{4}: \mathrm{H}_{2} \mathrm{O}: \mathrm{HNO}_{3}: \mathrm{CH}_{3} \mathrm{COOH}(16: 2: 1: 1 v / v)$. Prior to photoresist removal, a second layer of Al was thermally deposited. Subsequently, the substrate was sonicated in acetone for $30 \mathrm{~min}$ to facilitate photoresist lift-off, followed by another oxygen plasma cleaning step to remove adsorbed organic residue from the Al nanogaps.

\subsection{Nanowire Fabrication}

Glass substrates with Al nanogaps (described above) were loaded into a high vacuum evaporator and $20 \mathrm{~nm}$ of $\mathrm{Cr}$ film was thermally evaporated at deposition rate of $10 \AA / \mathrm{s}$. Subsequently, the aluminum layer was etched away (lifted off) after 2 min exposure to a $1 \mathrm{M}$ aqueous $\mathrm{KOH}$ solution, resulting $\mathrm{Cr}$ nanowires. For gold and silver nanowires, a thin layer of chromium (5 nm) was first deposited, followed by deposition of $40 \mathrm{~nm}$ gold or silver at a deposition rate of 10 and $20 \AA / \mathrm{s}$.

\subsection{Micropillar-Based Microfluidic Photomask Fabrication}

Three hundred (300) $\mathrm{nm}$ thermally evaporated $\mathrm{Cr}$ film was first deposited on glass substrates at a rate of $10 \AA / \mathrm{s}$. Subsequently, $1.5 \mu \mathrm{m}$ of S1813 photoresist was spin-coated on top of the $\mathrm{Cr}$ film and baked at $120{ }^{\circ} \mathrm{C}$ for $10 \mathrm{~min}$. Following UV exposure, development and stripping (using oxygen plasma), the exposed metal was over-etched for $15 \mathrm{~min}$ using a $\mathrm{Cr}$ etchant solution obtained from Sigma Aldrich composed of diammonium hexanitratocerate $(10 \%-30 \%)$ and nitric acid $(5 \%-10 \%)$ ). Following an aqueous wash and an oxygen plasma cleaning steps, an additional $300 \mathrm{~nm}$ thermally evaporated Cr film was deposited at a rate of $10 \AA / \mathrm{s}$. Subsequently, the S1813 photoresist was lifted off by sonicating in acetone for $30 \mathrm{~min}$ followed by another oxygen plasma cleaning step. The resulting microgap pattern was then adjusted to the desired length using another mask (defined as cutting mask). For this the microgap length was defined with a second photolithography step using S1813 photoresist that was spin-coated and pre-heated at $120{ }^{\circ} \mathrm{C}$ for $10 \mathrm{~min}$ (producing a average thickness of $1.5 \mu \mathrm{m}$ ) before exposing through a "cutting mask" that is placed perpendicular to the original microgap pattern. After UV exposure, photoresist development and $90 \mathrm{~s}$ Cr etch, the unexposed photoresist was stripped in acetone and cleaned in DI water and oxygen plasma. Subsequently, the resulting pattern was incorporated into the microfluidic channel using a third photolithographic step, similar as above (i.e., spin-coating of S1813 photoresist, UV-exposure, development and $300 \mathrm{~nm}$ thermal evaporation of $\mathrm{Cr}$ ) before sonication-assisted lift-off in acetone for $30 \mathrm{~min}$.

\subsection{Microfluidics Device Fabrication and Microbead Filtration}

A clean silicon ( $\mathrm{Si}$ ) wafer was spin-coated with $12 \mu \mathrm{m}$-thick of SU-8 3010. After pre-baking for $1 \mathrm{~min}$ at $65^{\circ} \mathrm{C}$ and $12 \mathrm{~min}$ at $95^{\circ} \mathrm{C}$, the SU-8 3010 film was exposed using the $\mathrm{Cr}$ photomask realized in Section 2.4. Following a 12 min post-bake step at $95{ }^{\circ} \mathrm{C}$, the SU-8 3010 was developed and further hard baked at $150{ }^{\circ} \mathrm{C}$ for $90 \mathrm{~min}$. Subsequently, PDMS pre-polymer and its corresponding curing agent were mixed at a 10:1 volume ratio and casted over the SU-8 mold. After curing at $90{ }^{\circ} \mathrm{C}$ for 90 min, PDMS was peeled off the mold and two access holes where punched out, via a blunt-end 23-gauge 
needle, to serve as liquid inlets and outlets. Both PDMS mold and glass slides were then washed with soap, rinsed thoroughly, air-dried, exposed to oxygen plasma (4 min), affixed together and then heated at $90{ }^{\circ} \mathrm{C}$ for $1 \mathrm{~h}$. Fluids were delivered and withdrawn from microfluidic devices using polyethylene (PE) tubes with inner diameter $0.28 \mathrm{~mm}$ that were inserted into the pre-punched inlet and outlet holes. The desired fluid flow was established using a syringe pump, connected to the outlet and the microfluidic device and operated in withdraw mode.

\section{Results and Discussion}

Recent advances in high-resolution inkjet and laser printing have enabled the facile production of plastic masks at a fraction of the cost of high-end chrome analogues. The only drawback of plastic masks is that the smallest feature clearly definable is 25 microns. By extending the "edge-patterning" method of Whitesides et al. [32] we herein investigate the ability to use plastic masks for the formation of: (i) nanowires; (ii) sub-micron photo masks; and (iii) high-aspect ratio micropillar filters.

\subsection{Nanogaps and Nanowires}

Figure 1 illustrates the fabrication of nanogaps and nanowires based on "edge-patterning" of a thin aluminum film. The process starts with deposition of $100 \mathrm{~nm} \mathrm{Al}$ via thermal evaporation or other deposition techniques on a substrate. The choice of substrates can vary widely from glass, silicon or other insulating, semiconducting or metallic substrates, whose work functions are substantially higher than that of Al. Subsequently, a thick positive photoresist layer (i.e., $1.8 \mu \mathrm{m}$ ) was spin-coated onto the Al layer and patterned via UV lithography through a plastic photomask. After photoresist development, (Figure 1a) the exposed Al pattern was dissolved using a 16:2:1:1 v/v mixture of $\mathrm{H}_{3} \mathrm{PO}_{4}: \mathrm{H}_{2} \mathrm{O}: \mathrm{HNO}_{3}: \mathrm{CH}_{3} \mathrm{COOH}$. This ratio allows for slow $\mathrm{Al}$ etching that not only dissolves the exposed metal but also gradually etches the aluminum underneath the photoresist (Figure 1b). As described below, the width of the $\mathrm{Al}$ undercutting at the photoresist edge can be controlled depending on the exposure time to the aluminum etchant. Subsequent deposition of a second Al layer (green, Figure 1c), followed by acetone-assisted photoresist lift-off (Figure 1d) produces a gap, that depending on etching time and to a lesser extent on the granular size of Al, it can vary from nanometer size to several microns. Such gaps have the exact shape of the photoresist edge that was originally patterned on the substrate (Figure 1a).

Figure 2a demonstrates typical Al nanogaps realized by edge patterning method. Nanogaps with width of $c a$. $160 \pm 13 \mathrm{~nm}$ are obtained by exposing a thin $(50 \mathrm{~nm})$ Al layer for $175 \mathrm{~s}$ to the aforementioned $\mathrm{H}_{3} \mathrm{PO}_{4}: \mathrm{H}_{2} \mathrm{O}: \mathrm{HNO}_{3}: \mathrm{CH}_{3} \mathrm{COOH}$ etchant mixture. Such nanogaps are relatively uniform and continuous along the entire perimeter of the photolithographed object. The resulting Al nanogap can then be used as a lift-off layer to pattern various types of nanowires (NWs) as illustrated in Figure 1e,f. For this, a variety of metals (i.e., $\mathrm{Au}, \mathrm{Ag}, \mathrm{Pd}, \mathrm{Cr}, \mathrm{Ti}, \mathrm{Ni}$, etc.) and semiconductors (i.e., $\mathrm{CdSe}, \mathrm{CdS}, \mathrm{TiO}_{2}, \mathrm{ZnO}$, $\mathrm{In}_{2} \mathrm{O}_{3}$ and $\mathrm{SnO}_{2}$ etc.) can be deposited via thermal evaporation, sputtering, e-beam or chemical vapor deposition (CVD) followed by an Al lift-off step. Such Al lift-off (Figure 1f) is realized in either acidic (i.e., $\mathrm{H}_{3} \mathrm{PO}_{4}: \mathrm{H}_{2} \mathrm{O}: \mathrm{HNO}_{3}: \mathrm{CH}_{3} \mathrm{COOH}$ etchant mixture) or basic $(1 \mathrm{M} \mathrm{KOH})$ media, depending on the compatibility of metal or metal oxide based nanowires and etching solution. When compared to lithographically patterned nanowire electro-deposition (LPNE) method that was reported by 
Penner et al. [37,40], the edge-patterning method described herein offer a highly flexible venue to realize nanowire architectures. Moreover, unlike LPNE that requires conductive surfaces and environmental unfriendly metal precursors, Al lift-off is compatible with all substrates and common acids and bases.

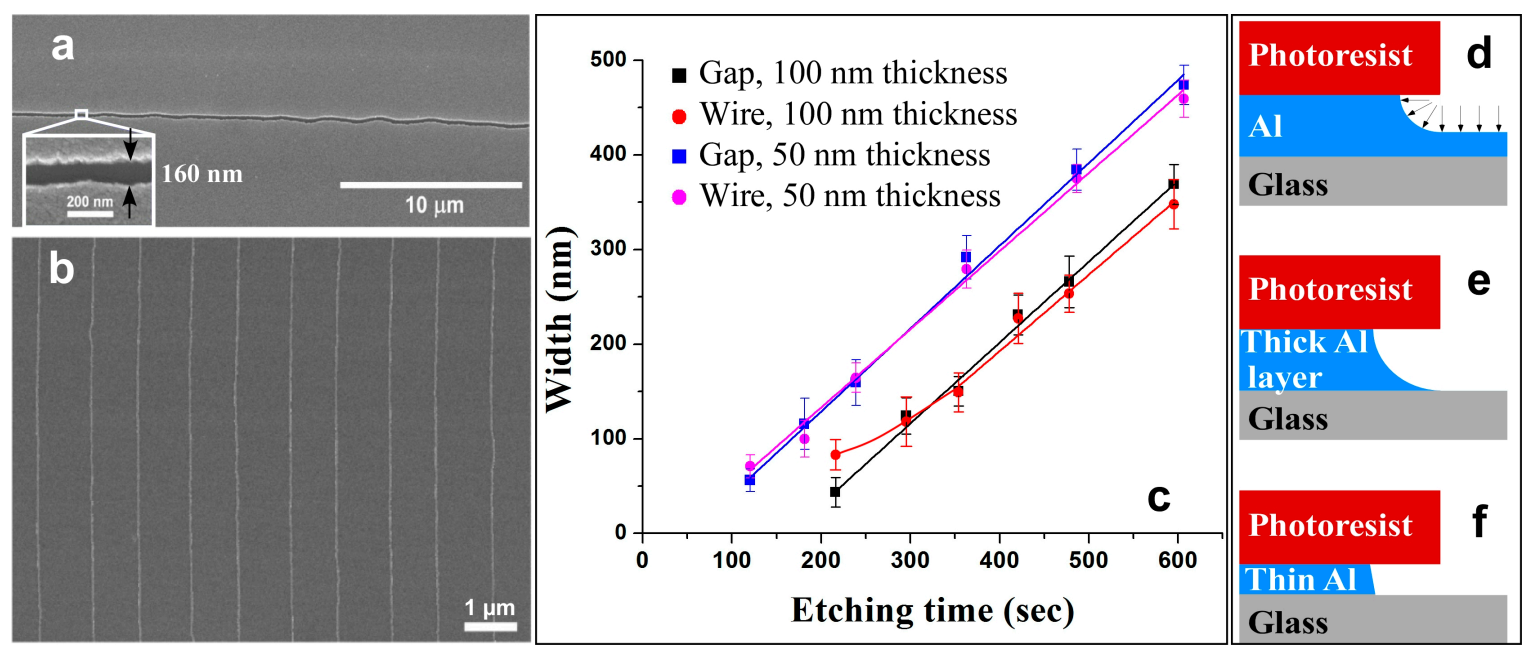

Figure 2. (a) SEM images of Al nanogaps formed using the process described in Figure 1a-d; (b) Representative $\mathrm{Cr}$ nanowires realized by thermal evaporation of chromium onto the Al-nanogaps of (a) and Al lift-off (shown in Figure 1e,f); (c) Nanogap and nanowire width for two Al thickness layers, as a function of Al etching time. The Al undercut produced by isotropic etching (d) works less optimally for thick (e); as opposed to thin (f) Al layers and small etching times.

To estimate the minimum width of nanowires that Al lift-off process can produce, the effect of etching time was studied. Figure 2c illustrates both nanogap and nanowire widths as a function of etching time for two Al layer thicknesses (i.e., 100 and $50 \mathrm{~nm}$ ). Similar etching rates $(c a .0 .91 \mathrm{~nm} / \mathrm{s}$ ) were obtained for both thick and thin Al layers, indicating that the rate limiting step is 1-D diffusion of etching reagents and soluble byproducts across the undercut. In general, a near 1-to-1 correspondence is observed between the nanogap width and nanowire diameter [37]. For thick Al layers and small etching times, however, the system deviates from good replication. For example, when the nanogap approaches the thickness of the Al layer, the nanogaps show a significantly rougher edge and large number of discontinuities (not shown), with the nanowire significantly larger (i.e., $(85 \pm 16 \mathrm{~nm})$ than that of the gap (i.e., $42 \pm 15 \mathrm{~nm}$ ). This is due to the nature of isotropic wet etching (Figure $2 \mathrm{~d}$ ), which leaves a thin Al layer on glass surface and is also responsible for: (a) increasing the average nanowire thickness (shown by the departure of red curve from the black curve in Figure 2c, and (b) decreasing the adhesion between the nanowire and glass surface. Correspondingly, the profile of the thin Al layer (i.e., $50 \mathrm{~nm}$ ) becomes much sharper than that of thick Al layer (i.e., $100 \mathrm{~nm}$ ), as shown in Figures 2c,e,f [41]. Using $50 \mathrm{~nm}$ thin layers, continuous nanogap and nanowires were realized around various patterns. Typically, the thinnest continuous nanogap obtained was $c a .50 \pm 10 \mathrm{~nm}$, while the corresponding nanowire was slightly larger at $c a .70 \pm 20 \mathrm{~nm}$ (Figure $2 \mathrm{c}$ ).

To demonstrate the continuity of these nanowires over large distances (up to $20 \mathrm{~mm}$ in length), we tested their current-voltage (I-V) characteristics using a simple two-point-probe method. For this, 
two $\mathrm{Cr}$ contacting pads were patterned at the ends of the nanowires (Figure 3a) via spin-coating positive photoresist and using another plastic mask to photolithographically pattern contacts at the desired nanowire length (L) (Figure 3a). Figure $3 \mathrm{~b}$ illustrates that the electrical resistance of $\mathrm{Ag}$ nanowires with $43 \pm 2 \mathrm{~nm}$ in thickness and $300 \mathrm{~nm}$ in width varies linearly with their length (L) (i.e., from 12 to $1000 \mu \mathrm{m}$ ). This indicates the structure uniformity of these nanowires over long lengths, with no significant defects presented. To demonstrate the uniformity that the Al lift off approach affords in making these nanowires, Cr contacts were deposited onto two 43 by $300 \mathrm{~nm}$ nanowires with that were $500 \mu \mathrm{m}$ spaced apart. As shown in Figure 3c, the conductance of both nanowires can be measured first and subsequently, one nanowire can be selectively severed with a razor blade scratch. The conductance of two $500 \mu \mathrm{m}$ long nanowires is $690 \mu \mathrm{S}$, while the single nanowire conductance was nearly half (i.e., $310 \mu \mathrm{S}$ ), which is within $10 \%$ error. In order to get a better estimate of nanowire uniformity, the resistivity of six pairs of 43 by $400 \mathrm{~nm} \mathrm{Ag}$ nanowires with length of $2 \mathrm{~cm}(20,000 \mu \mathrm{m})$ (Figure 3a) were measured using 2-point probe. The insert table in Figure 3a shows that the resistance of the six nanowire pairs is fairly uniform, averaging $43 \mathrm{k} \Omega$ and with standard deviation of $18 \%$. Both aforementioned long and short nanowire resistivity tests indicate that the Al lift-off method is capable of preparing continuous, defect-free nanowires with good electrical uniformity, that can easily extend from a couple to tens of thousands of microns in length.

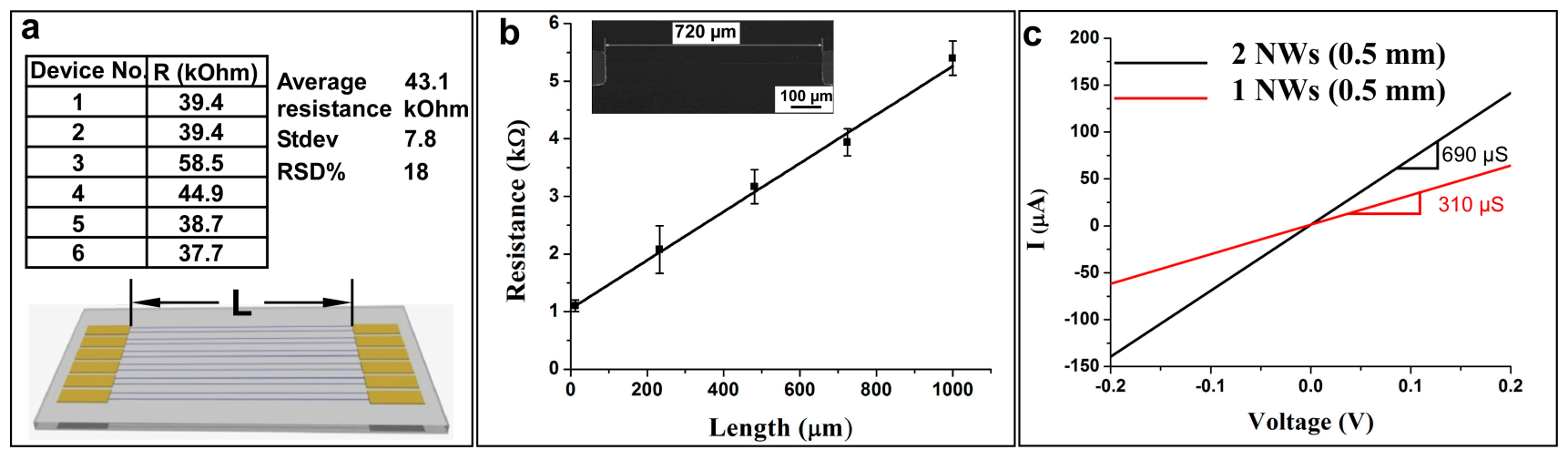

Figure 3. (a) Six pairs of silver nanowires with $\mathrm{Cr}$ contact pads at their ends. Insert table shows the electrical resistance values of six pairs of $43 \mathrm{~nm}$ thick, $400 \mathrm{~nm}$ wide and $2 \mathrm{~cm}$ $(20,000 \mu \mathrm{m})$ long Ag nanowires; (b) 2-point probe electrical resistance of a Ag nanowire (with $43 \mathrm{~nm}$ in thickness and $300 \mathrm{~nm}$ in width) as a function of nanowire length. Inset in (b) shows the SEM image of a $720 \mu \mathrm{m}$ long nanowire with its $\mathrm{Cr}$ contact pads; (c) I-V characteristics of one (red) and two (black) Ag nanowire(s) with $43 \mathrm{~nm}$ in thickness, $300 \mathrm{~nm}$ in width and $500 \mu \mathrm{m}$ in length.

\subsection{Micropillar-Based Filtration within Microfluidic Devices}

The low cost and high fidelity of the Al lift-off method described above can not only be used to realize nanowire arrays but also assist in incorporating micropillar-based filtration within microfluidic devices. Such micropillar-filtration could be designed for on-line separation of large objects (i.e., microspheres, cells, aggregates, etc.) within low-cost microfluidic devices for point-of-care diagnostics. With this in mind, we tested the applicability of the "edge-patterning" method [32] in assisting to localize $6 \mu \mathrm{m}$ of beads within a confined area of a microfluidic device. 
Figure 4 illustrates the key steps to incorporate micron- (and ultimately sub-micron) gaps within a mask that can be used to fabricate the master (mold) for PDMS-based microfluidic channel. For this, $\mathrm{Cr}$ is substituted for the Al layer of Figure 1a, and used in "edge-patterning" to produce micron-sized gaps. These Cr gaps allow the formation of cheap, high-resolution photomasks for optical patterning of continuous stripes with edge-fidelity comparable to high-end photomasks [42,43]. For this, $300 \mathrm{~nm}$ thermally evaporated $\mathrm{Cr}$ film was deposited on a glass substrate. After positive photoresist patterning using a plastic mask with rectangular 25 by $500 \mu \mathrm{m}$ features that were spaced apart by $25 \mu \mathrm{m}$ gaps, the $\mathrm{Cr}$ was etched using a commercial diammonium-hexanitratocerate/nitric acid mixture (see experimental) for $15 \mathrm{~min}$ to produce a $2 \mu \mathrm{m}$ undercut beneath the photoresist (Figure 1b). Subsequently, another $300 \mathrm{~nm}$ thermally evaporated Cr film was deposited (Figure 1c and lifted off (Figure 1d) to produce a continuous $2 \mu \mathrm{m}$ gaps around the edge of the rectangular 25 by $1000 \mu \mathrm{m}$ features. In order to transform these rectangular $\mathrm{Cr}$ gaps into an array of $\mathrm{Cr}$ lines shown in Figure $4 \mathrm{a}$, their tops and bottoms together as well as their surrounding $\mathrm{Cr}$ must be removed. This was realized by patterning positive photoresist across the rectangular Cr gaps with a width (L) as shown in both Figures 3a and 4a. Following $\mathrm{Cr}$ etch and photoresist strip-off, an array of $2 \mu \mathrm{m} \mathrm{Cr}$ gaps is realized on top of glass substrate (Figure 4a). Figure 4b-f illustrate the subsequent steps in order to insert such array of $2 \mu \mathrm{m}$ $\mathrm{Cr}$ gaps within a much larger microfluidic channel. In brief, positive photoresist was spun on top of the $\mathrm{Cr}$ gap array (Figure 4b) and then roughly aligned with of microfluidic channel plastic photomask (Figure 4c). Following UV-exposure and photoresist development (Figure 4d) a third layer of $\mathrm{Cr}(300 \mathrm{~nm})$ is thermally evaporated (Figure 4e) and lifted off to produce a homemade micropillar filter incorporated within a microfluidic channel. In such fashion, the size, shape and length of the microgaps can be readily adjusted in order to vary and eventual optimize the performance of the microfluidic filtration device described below.
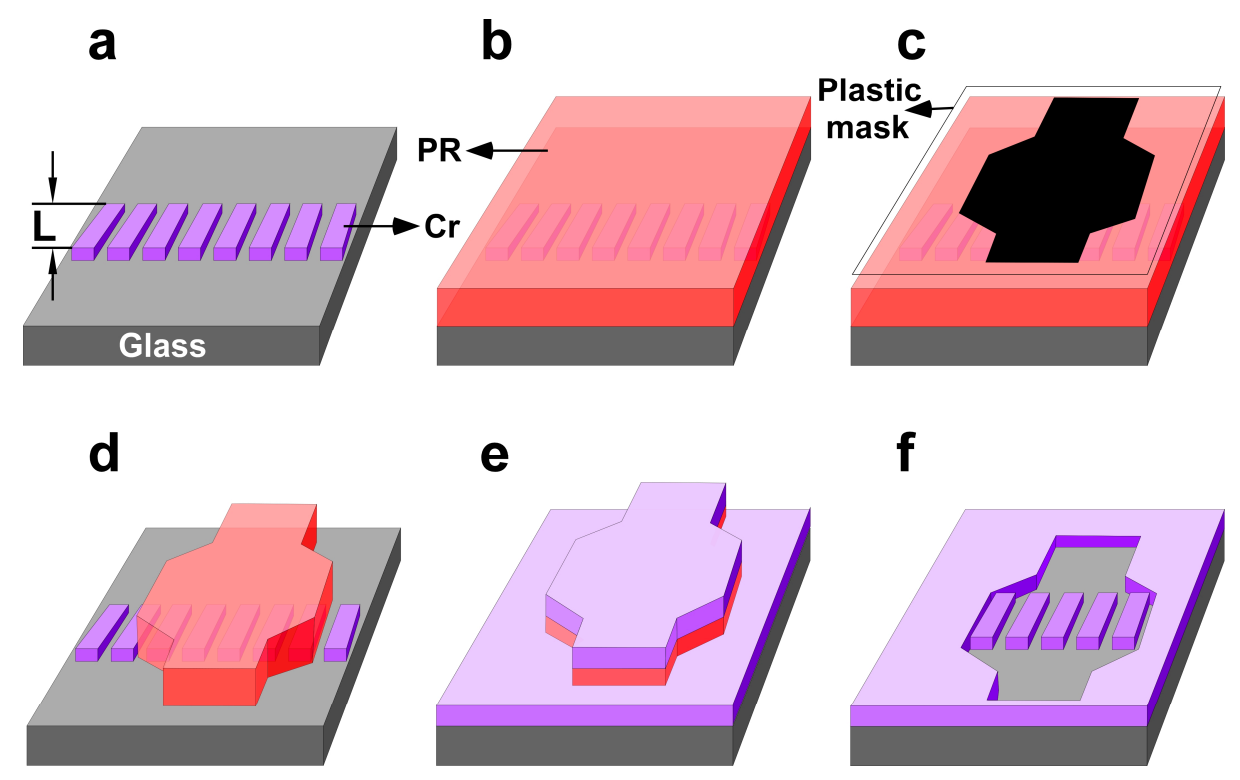

Figure 4. Incorporation of the array of microgaps (a) into a photomask to produce microfiltration masters for PDMS microfluidics. Following positive photoresit spin coating (b), photo-patterning with a plastic mask that defines the microfluidic channel (c) protects the microgaps (d) from Cr filling (e) until lift-off (f), which completes the formation of a high resolution, homemade, microfiltration photo-mask. 
Figure 5 illustrates optical and SEM micrographs of the high resolution, custom-made, microfiltration photo-mask, whose fabrication is described above. As shown in Figure 5a, $2.2 \mu \mathrm{m}$ continuous $\mathrm{Cr}$ gaps are realized, despite the edge irregularities and roughness of a lesser quality, rectangular starting plastic photomask. Despite the roughness and irregularities the edge-patterning method affords the formation of continuous channels devoid of any blockage. Figure $5 \mathrm{~b}$ depicts the incorporation of the array of $\mathrm{Cr}$ gaps within a microfluidic channel (i.e., top and bottow black gaps). The widened filtration region with respect to the inlet and outlet (shown on top of Figure 5b) of the regular microfluidic channel is designed in order to account for the flow resistance through the microfiltration array.

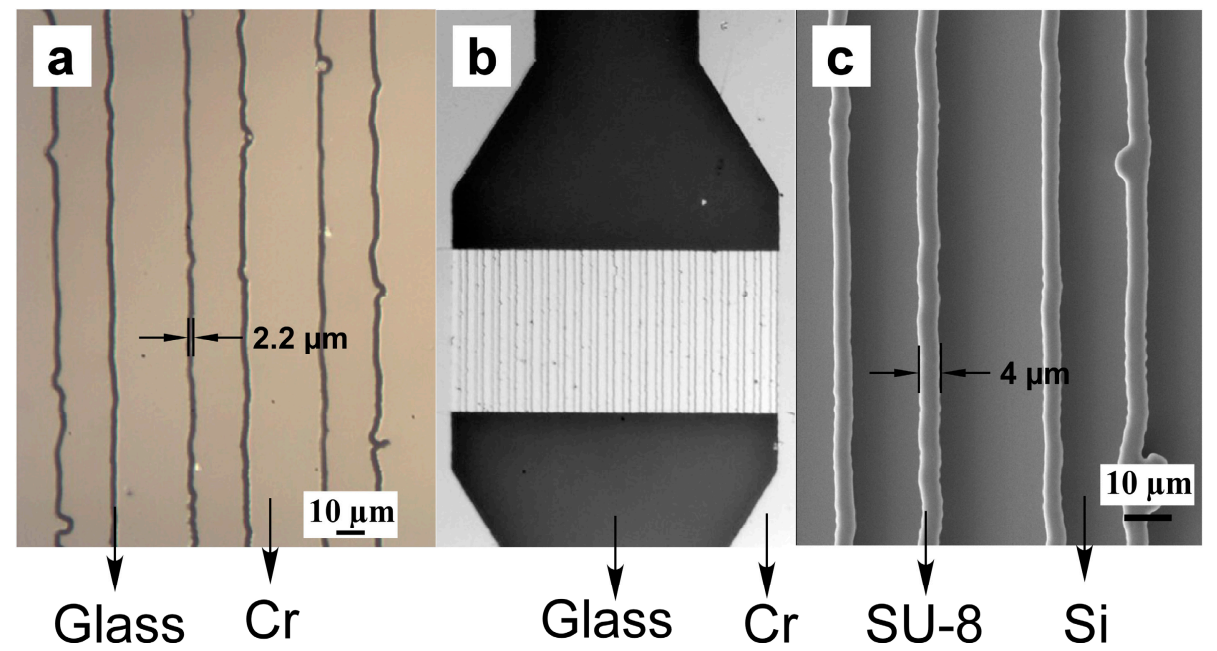

Figure 5. Optical and SEM micrographs of the homemade Cr mask utilized to form an array of $\mathrm{Cr}$ microgaps (a) within a microfluidic channel (b). This mask is then used to photolithographically pattern SU-8 photoresist in order to realize an array of rectangular-shaped micro-pillars (c).

The homemade mask (Figure 5b) was then used to realize masters for the production of PDMS microfluidics. For this a negative SU-8 3010 photoresist was spin coated on Si wafer with thickness of $c a .10 \mu \mathrm{m}$ after 1 and $10 \mathrm{~min}$ pre-baking at 65 and $95{ }^{\circ} \mathrm{C}$, respectively. Following $0.8 \mathrm{~s} \mathrm{UV}$ exposure (with UV light intensity ca. $30 \mathrm{~mW} / \mathrm{cm}^{2}$ ) and $12 \mathrm{~min}$ post-bake at $95{ }^{\circ} \mathrm{C}$, the substrate was soaked in SU8 developer for 6 min and washed thoroughly with additional SU8 developer and isopropanol before being dried with air. Figure 5c depicts an SEM micrograph of the resulting SU8 rectangular micropillars with an average width and height of 4.0 and $10 \mu \mathrm{m}$, respectively (i.e., cross-sectional aspect ratio of 2.5). Such micropillars are continuous and closely trace the edge roughness of the homemade Cr mask. The nearly double width of SU-8 micropillars compared to that of the gaps of the homemade Cr mask is attributed to edge diffraction effects [44,45] The use of SU-8 3010 as opposed to SU-8 2010 and others was chosen because of its reported higher adhesion to Si wafers. This is important to ensure the continuity of SU-8 micropillars and their resistance against delaminating from their Si substrates following the PDMS removal (described below).

The targeted microfluidic device was fabricated by casting the mixture of PDMS prepolymer and crosslinking agent over the aforementioned SU-8 3010 micropillars. Following vacuum treatment to remove all dissolved air and bubbles, the mixture is heated on a hot plate at $90{ }^{\circ} \mathrm{C}$ for $2 \mathrm{~h}$, allowed to cool down at room temperature and then peeled-off. Figure $6 \mathrm{a}, \mathrm{b}$ illustrate the cross-sectional view of 
the micropillar gaps. The bottom width of micropillar gaps closely tracks the SU-8 3010 micropillar master. At the other end, the gap shrinks by $c a$. $25 \%$ to $3 \mu \mathrm{m}$, as shown clearly in Figure $6 \mathrm{~b}$. Such unexpected size reduction, is attributed to the elastic nature of PDMS that improves the cross sectional aspect ratio from 2.5 (of the master) to 3 (for the mold). Following needle punching of an inlet and outlet at each end of the microfluidic channel, the PDMS was plasma-treated together with the glass slide to which it was later affixed by heating at $90{ }^{\circ} \mathrm{C}$ for $1 \mathrm{~h}$ to afford an irreversible bond between the two [46]. Subsequently, a dilute mixture of $5.8 \mu \mathrm{m}$ microbeads with bead concentration ranging from $0.001 \mathrm{mg} / \mathrm{mL}$ to $5 \mathrm{mg} / \mathrm{mL}$ in phosphate buffer saline (PBS) was introduced into the microfluidic channel using a needle-assisted suction from the other end. As shown in Figure $6 \mathrm{c}$,d, as well as the video in the Supplementary Information section, the aforementioned micropillar gap device can effectively stop the passage of these microbeads, without blocking the fluid flow. This is attributed to the fact that the channel height cannot tolerate two microbeads on top of each other (otherwise the 2nd layer would have come in focus in the video, when intentionally defocusing away of the 1st layer), therefore allowing the uninterrupted flow of PBS, while no microbeads managed to traverse the micropillar gap barrier. This demonstrates the viability of the micropillar gap filtration method using low-cost home-made masks with traditional UV-photolithography, thereby negating the need of expensive commercial masks [25] and expensive deep-reactive ion etch (DRIE) masters [22].
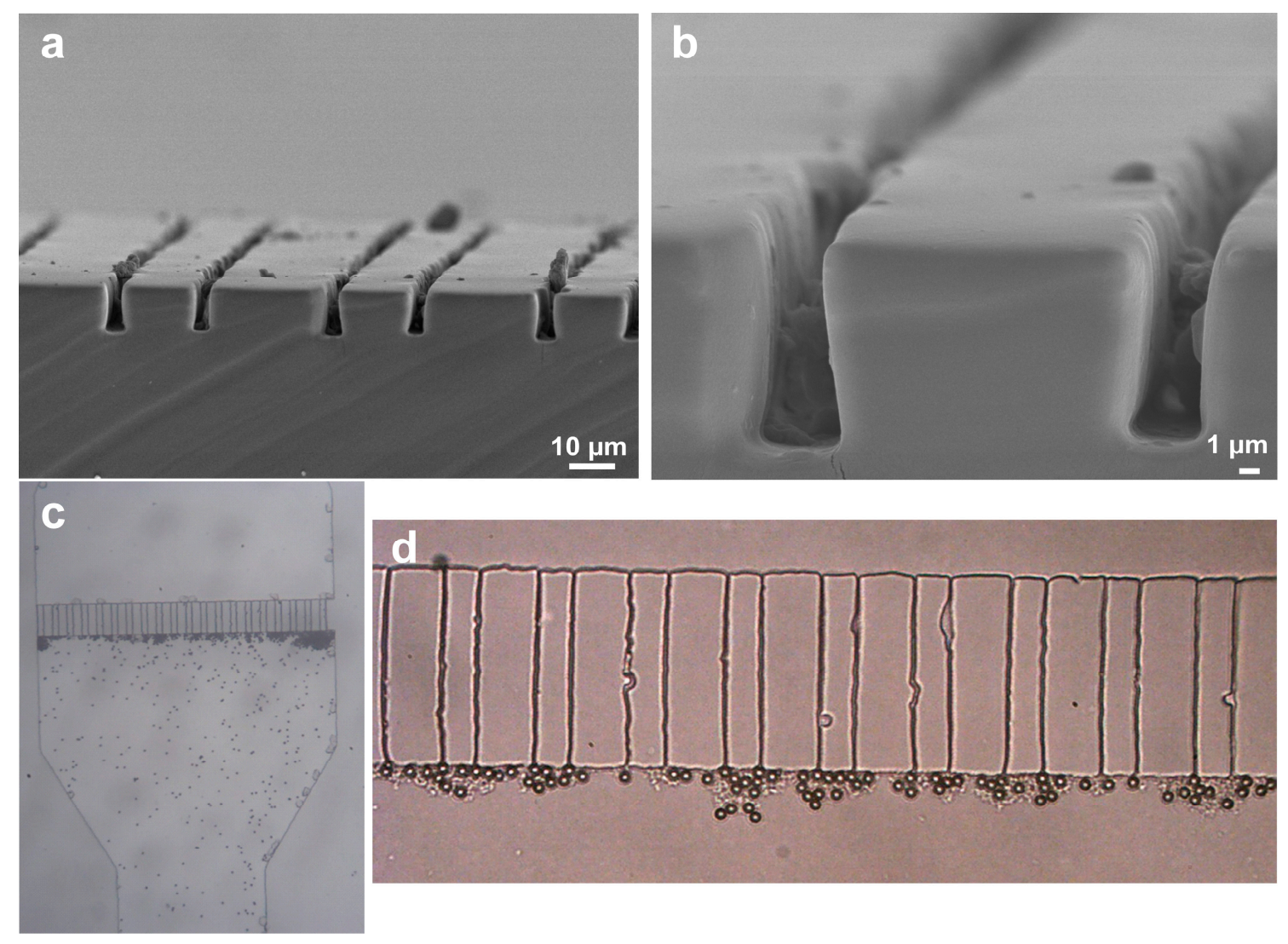

Figure 6. PDMS microfluidic devices that incorporate micropillar-based filters, obtained from homemade Cr-made mask and SU-8 3010 masters shown in Figure 5. (a) and (b) illustrate SEM cross-sections of the PDMS micropillar filters. (c) and (d) show optical micrographs of PDMS-incorporated micropillar filters to arrest the flow of $6 \mu \mathrm{m}$ beads, while allowing the rest of the solution to flow through. 


\subsection{Conclusions and Outlook}

The work described expands on the use of edge-patterning [32] based on isotropic wet etching underneath a patterned photoresist to define nano- to micron-sized gaps, wires and high-aspect ratio pillars. The height and width of these features depend on the thickness of the sacrificial low-work function metal (i.e., Al) and etching duration, respectively. Using the Al edge-patterning method, the formation of continuous and highly uniform $\mathrm{Cr}$ nanowire arrays was demonstrated with height and thicknesses of few tens and hundreds of nm, respectively, while their length spans to $\mathrm{cm}$ in size and electrical uniformity ranging from $10 \%$ to $20 \%$. Such patterning can be extended to a variety of high work function metals and semiconductors and further enrich nanowire architectures. Moreover, we show that edge-patterning can be translated from $\mathrm{Al}$ to $\mathrm{Cr}$, thereby enabling its use for the formation of high resolution, custom-made $\mathrm{Cr}$ masks which are constructed from relatively inexpensive plastic masks. These custom-made $\mathrm{Cr}$ masks are ideal for patterning high-aspect ratio features for electronic, photonic, and biosensor based applications. Using this concept, we produced micro-pillar SU-8 masters and corresponding PDMS microfluidic molds with 3-4 $\mu \mathrm{m}$ gaps, suitable for filtering out $6 \mu \mathrm{m}$ beads. The ability to spatially define nanowire arrays and microgap filters may offer a large advantage for automated microfluidic electrochemical immunoassays where microelectrode 3D diffusion and sample pre-concentration can play a crucial role for signal amplification. In general, the approach described herein may facilitate the integration of microfluidic devices with a variety of nano/micro concepts to improve detection limits and lower costs for the eventual development of cheap point-of-care medical diagnostics based on detection of molecular biomarkers in human body fluids.

\section{Acknowledgments}

The authors wish to acknowledge financial support by the NIH RO1EB014586 and partial support by US-Army TATRC W81XWH-09-1-0711 grants.

\section{Author Contributions}

N.M.D fabricated homemade masks, SU-8 based mold, and PDMS microfluidic devices. L.Q. fabricated and characterized the Ag nanowires. Z.L. and S.V. assisted L.Q. and N.M.D with mask fabrication. G.W.B. and J.F.R. assisted N.M.D. with microbead suspension and microfiltration. F.P. supervised this work and assisted N.M.D. and L.Q. with manuscript drafting.

\section{Supplemental Information}

A micro-filtration video illustrating that the PDMS micropillar array is capable to retain and concentrate a $2.5 \mathrm{mg} / \mathrm{mL}$ dispersion of $6 \mu \mathrm{m}$ micro-beads, while the suspending fluid (10 mM PBS) flows smoothly through.

\section{Conflict of Interest}

The authors declare no conflict of interest. 


\section{References}

1. Erickson, D.; Li, D. Integrated microfluidic devices. Anal. Chim. Acta 2004, 507, 11-26.

2. Han, K.N.; Li, C.A.; Seong, G.H. Microfluidic chips for immunoassays. Annu. Rev. Anal. Chem. (Palo Alto, Calif.) 2013, 6, 119-141.

3. Ojeda, I.; Moreno-Guzman, M.; Gonzalez-Cortes, A.; Yanez-Sedeno, P.; Pingarron, J.M. Electrochemical magnetoimmunosensor for the ultrasensitive determination of interleukin-6 in saliva and urine using poly-hrp streptavidin conjugates as labels for signal amplification. Anal. Bioanal. Chem. 2014, 406, 6363-6371.

4. Shamsi, M.H.; Choi, K.; Ng, A.H.C.; Wheeler, A.R. A digital microfluidic electrochemical immunoassay. Lab Chip 2014, 14, 547-554.

5. Díaz-González, M.; Baldi, A. Fabrication of biofunctionalized microfluidic structures by low-temperature wax bonding. Anal. Chem. 2012, 84, 7838-7844.

6. Otieno, B.A.; Krause, C.E.; Latus, A.; Chikkaveeraiah, B.V.; Faria, R.C.; Rusling, J.F. On-line protein capture on magnetic beads for ultrasensitive microfluidic immunoassays of cancer biomarkers. Biosens. Bioelectron. 2014, 53, 268-274.

7. Tang, C.K.; Vaze, A.; Rusling, J.F. Fabrication of immunosensor microwell arrays from gold compact discs for detection of cancer biomarker proteins. Lab Chip 2012, 12, 281-286.

8. Rusling, J.F.; Bishop, G.W.; Doan, N.M.; Papadimitrakopoulos, F. Nanomaterials and biomaterials in electrochemical arrays for protein detection. J. Mater. Chem. B 2014, 2, 12-30.

9. Sardesai, N.; Kadimisetty, K.; Faria, R.; Rusling, J. A microfluidic electrochemiluminescent device for detecting cancer biomarker proteins. Anal. Bioanal. Chem. 2013, 405, 3831-3838.

10. Fan, F.; Shen, H.; Zhang, G.; Jiang, X.; Kang, X. Chemiluminescence immunoassay based on microfluidic chips for $\alpha$-fetoprotein. Clin. Chim. Acta 2014, 431, 113-117.

11. Ge, L.; Yan, J.; Song, X.; Yan, M.; Ge, S.; Yu, J. Three-dimensional paper-based electrochemiluminescence immunodevice for multiplexed measurement of biomarkers and point-of-care testing. Biomaterials 2012, 33, 1024-1031.

12. Venkatanarayanan, A.; Crowley, K.; Lestini, E.; Keyes, T.E.; Rusling, J.F.; Forster, R.J. High sensitivity carbon nanotube based electrochemiluminescence sensor array. Biosens. Bioelectron. 2012, 31, 233-239.

13. Justino, C.I.L.; Freitas, A.C.; Amaral, J.P.; Rocha-Santos, T.A.P.; Cardoso, S.; Duarte, A.C. Disposable immunosensors for c-reactive protein based on carbon nanotubes field effect transistors. Talanta 2013, 108, 165-170.

14. Kamahori, M.; Ishige, Y.; Shimoda, M. Enzyme immunoassay using a reusable extended-gate field-effect-transistor sensor with a ferrocenylalkanethiol-modified gold electrode. Anal. Sci. 2008, 24, 1073-1079.

15. Li, J.; Zhang, Y.; To, S.; You, L.; Sun, Y. Effect of nanowire number, diameter, and doping density on nano-fet biosensor sensitivity. ACS Nano 2011, 5, 6661-6668.

16. Makowski, M.S.; Ivanisevic, A. Molecular analysis of blood with micro-/nanoscale field-effect-transistor biosensors. Small 2011, 7, 1863-1875.

17. Patolsky, F.; Zheng, G.; Lieber, C.M. Nanowire-based biosensors. Anal. Chem. 2006, 78, 4260-4269. 
18. Tkac, J.J.D. Engineering the Bioelectronic Interface; Royal Society of Chemistry: Philadelphia, PA, USA, 2009; pp. 193-224.

19. Ikami, M.; Kawakami, A.; Kakuta, M.; Okamoto, Y.; Kaji, N.; Tokeshi, M.; Baba, Y. Immuno-pillar chip: A new platform for rapid and easy-to-use immunoassay. Lab Chip 2010, 10, 3335-3340.

20. Mayer, K.M.; Lee, S.; Liao, H.; Rostro, B.C.; Fuentes, A.; Scully, P.T.; Nehl, C.L.; Hafner, J.H. A label-free immunoassay based upon localized surface plasmon resonance of gold nanorods. ACS Nano 2008, 2, 687-692.

21. Chin, C.D.; Laksanasopin, T.; Cheung, Y.K.; Steinmiller, D.; Linder, V.; Parsa, H.; Wang, J.; Moore, H.; Rouse, R.; Umviligihozo, G.; et al. Microfluidics-based diagnostics of infectious diseases in the developing world. Nat. Med. 2011, 17, 1015-1015.

22. Andersson, H.; Van Der Wijngaart, W.; Enoksson, P.; Stemme, G. Micromachined flow-through filter-chamber for chemical reactions on beads. Sens. Actuators B Chem. 2000, 67, 203-208.

23. Maeng, J.-H.; Lee, B.-C.; Ko, Y.-J.; Cho, W.; Ahn, Y.; Cho, N.-G.; Lee, S.-H.; Hwang, S.Y. A novel microfluidic biosensor based on an electrical detection system for alpha-fetoprotein. Biosens. Bioelectron. 2008, 23, 1319-1325.

24. Chen, X.; Cui, D.; Chen, J. Design, fabrication and characterization of nano-filters in silicon microfluidic channels based on mems technology. Electrophoresis 2009, 30, 3168-3173.

25. Ruffert, C.; Ramadan, Q.; Gijs, M.A.M. Fabrication of a high aspect ratio (har) micropillar filter for a magnetic bead-based immunoassay. Microsyst. Technol. 2014, 20, 1869-1873.

26. Fu, Z.; Shao, G.; Wang, J.; Lu, D.; Wang, W.; Lin, Y. Microfabricated renewable beads-trapping/releasing flow cell for rapid antigen-antibody reaction in chemiluminescent immunoassay. Anal. Chem. 2011, 83, 2685-2690.

27. Vieu, C.; Carcenac, F.; Pépin, A.; Chen, Y.; Mejias, M.; Lebib, A.; Manin-Ferlazzo, L.; Couraud, L.; Launois, H. Electron beam lithography: Resolution limits and applications. Appl. Surf. Sci. 2000, 164, 111-117.

28. Sandison, M.E.; Cooper, J.M. Nanofabrication of electrode arrays by electron-beam and nanoimprint lithographies. Lab Chip 2006, 6, 1020-1025.

29. Altissimo, M. E-beam lithography for micro-/nanofabrication. Biomicrofluidics 2010, 4, 026503.

30. Yan, X.M.; Kwon, S.; Contreras, A.M.; Bokor, J.; Somorjai, G.A. Fabrication of large number density platinum nanowire arrays by size reduction lithography and nanoimprint lithography. Nano Lett. 2005, 5, 745-748.

31. Zhang, H.; Chung, S.W.; Mirkin, C.A. Fabrication of sub-50-nm solid-state nanostructures on the basis of dip-pen nanolithography. Nano Lett. 2003, 3, 43-45.

32. Love, J.C.; Paul, K.E.; Whitesides, G.M. Fabrication of nanometer-scale features by controlled isotropic wet chemical etching. Adv. Mater. 2001, 13, 604-608.

33. Mani, V.; Chikkaveeraiah, B.V.; Patel, V.; Gutkind, J.S.; Rusling, J.F. Ultrasensitive immunosensor for cancer biomarker proteins using gold nanoparticle film electrodes and multienzyme-particle amplification. ACS Nano 2009, 3, 585-594.

34. Tennico, Y.H.; Hutanu, D.; Koesdjojo, M.T.; Bartel, C.M.; Remcho, V.T. On-chip aptamer-based sandwich assay for thrombin detection employing magnetic beads and quantum dots. Anal. Chem. 2010, 82, 5591-5597. 
35. Kim, D.N.; Lee, Y.; Koh, W.G. Fabrication of microfluidic devices incorporating bead-based reaction and microarray-based detection system for enzymatic assay. Sens. Actuators B Chem. 2009, 137, 305-312.

36. Mani, V.; Wasalathanthri, D.P.; Joshi, A.A.; Kumar, C.V.; Rusling, J.F. Highly efficient binding of paramagnetic beads bioconjugated with 100,000 or more antibodies to protein-coated surfaces. Anal. Chem. 2012, 84, 10485-10491.

37. Menke, E.J.; Thompson, M.A.; Xiang, C.; Yang, L.C.; Penner, R.M. Lithographically patterned nanowire electrodeposition. Nat. Mater. 2006, 5, 914-919.

38. Henstridge, M.C.; Compton, R.G. Mass transport to micro- and nanoelectrodes and their arrays: A review. Chem. Rec. (New York, N.Y.) 2012, 12, 63-71.

39. Rusling, J.F. Multiplexed electrochemical protein detection and translation to personalized cancer diagnostics. Anal. Chem. 2013, 85, 5304-5310.

40. Xiang, C.; Kung, S.-C.; Taggart, D.K.; Yang, F.; Thompson, M.A.; Guell, A.G.; Yang, Y.; Penner, R.M. Lithographically patterned nanowire electrodeposition: A method for patterning electrically continuous metal nanowires on dielectrics. ACS Nano 2008, 2, 1939-1949.

41. Ponoth, S.S.; Agarwal, N.T.; Persans, P.D.; Plawsky, J.L. Fabrication of controlled sidewall angles in thin films using isotropic etches. J. Vac. Sci. Technol. B Microelectron. Nanometer Struct. 2003, 21, 1240-1247.

42. Metev, S.M.; Vejko, V.P. Laser Assisted Microtechnology; Springer: Berlin, Germany, 1998.

43. Wu, H.; Odom, T.W.; Whitesides, G.M. Generation of chrome masks with micrometer-scale features using microlens lithography. Adv. Mater. 2002, 14, 1213-1216.

44. Ito, T.; Okazaki, S. Pushing the limits of lithography. Nature 2000, 406, 1027-1031.

45. Maluf, N.; Williams, K. Introduction to microelectromechanical systems engineering; Artech House, Inc.: Norwood, MA, USA, 2004.

46. Herold, K.E.; Rasooly, A. Lab on a Chip Technology, Volume 1; Caister Academic Press: Norfolk, UK, 2009.

(C) 2015 by the authors; licensee MDPI, Basel, Switzerland. This article is an open access article distributed under the terms and conditions of the Creative Commons Attribution license (http://creativecommons.org/licenses/by/4.0/). 